\begin{tabular}{|l|l|l|l|l|l|}
\hline MUNIBE Antropologia-Arkeologia & $n^{\circ} 70$ & $5-19$ & DONOSTIA & 2019 & ISSN 1132-2217 • eISSN 2172-4555 \\
\hline
\end{tabular}

\title{
El desarrollo de la investigación en los yacimientos Paleolíticos de Torralba y Ambrona (Soria, España) a partir de los diarios inéditos de Dolores Echaide
}

\author{
The development of the research in the Palaeolithic sites of Torralba and \\ Ambrona (Soria, Spain) based on the unpublished diaries of Dolores Echaide
}

PALABRAS CLAVES: Península Ibérica; Paleolítico antiguo; Achelense; Clark Howell; Historiografía.

GAKO-HITZAK: Iberiar penintsula; antzinako Paleolitoa; Acheulearra; Clark Howell; Historiografia.

KEY WORDS: Iberian Peninsula; Lower Palaeolithic; Acheulean; Clark Howell; Historiography.

Manuel SANTONJA(1)

\section{RESUMEN}

La arqueóloga Dolores Echaide (1928-2018) dedicó gran parte de su vida profesional a las localidades achelenses de Torralba y Ambrona, integrada en los equipos dirigidos por F. C. Howell en 1961-1963 y por Howell y L. G. Freeman en 1980-1983. Echaide contribuyó en cada campaña a la organización del trabajo e intervino de manera destacada en todos los procesos de excavación. La documentación elaborada por ella misma, recientemente depositada en el CENIEH, recoge numerosas observaciones que resultan esenciales para conocer en detalle las investigaciones realizadas en ambos yacimientos.

El proyecto iniciado en 1961 en Torralba responde a los principios que habían comenzado a adoptarse en yacimientos paleolíticos del Este de África, aplicando un concepto antropológico y multidisciplinar a su interpretación. Las campañas en Torralba y Ambrona contaron lamentablemente con escasa participación española, reducida en el campo arqueológico prácticamente a Dolores Echaide, y tuvieron muy poca repercusión en el desarrollo en España de la investigación del Paleolítico antiguo.

\section{LABURPENA}

Dolores Echaide (1928-2018) arkeologoak bere bizitza profesionaleko zati handi bat eman zuen Torralba eta Ambrona kokapen acheulearretan, 1961-1963an F. C. Howell-ek eta 1980-1983an L. G. Freeman-ek zuzendutako taldeetan. Echaidek lana antolatzen lagundu zuen kanpaina bakoitzean eta indusketa-prozesu guztietan esku-hartze handia izan zuen. Duela gutxi CENIEH Ikerketa-zentroan gorde duten eta Echaidek prestatu zuen dokumentazioak bi aztarnategi horietan egindako ikerketak xehetasun osoz ezagutzeko funtsezkoak diren behaketa ugari jasotzen du.

1961ean Torralban hasitako proiektuak Afrikako ekialdeko aztarnategi paleolitikoetan erabiltzen hasi ziren printzipioak biltzen ditu, interpretatzeko garaian kontzeptu antropologikoa eta diziplina anitzekoa aplikatuta. Zoritxarrez, ordea, Torralban eta Ambronan egindako kanpainetan espainiarren partaidetza txikia izan zen. Esparru arkeologikoari dagokionez, Dolores Echaidek bakarrik parte hartu zuela esan dezakegu eta horrek oihartzun txikia izan zuen antzinako Paleolitoaren ikerketak Espainian izandako garapenean.

\section{ABSTRACT}

The archaeologist Dolores Echaide (1928-2018) devoted much of her professional life to the excavation and research of the Acheulean sites of Torralba and Ambrona, integrated into the projects led by F. C. Howell in 1961-1963 and by Howell and L. G. Freeman in $1980-1983$. Echaide contributed fundamentally in each field season in the organization of work teams. She participated outstandingly recording the lithic and bone remains, planning and carrying out the stratigraphic control of the excavation processes, especially in Ambrona. Her diaries, as well as other documents that she produced -all of them recently deposited in the CENIEH- collect many notes which provide detailed information about the investigations carried out in both sites.

The project started in 1961 in Torralba responds to the same principles that researchers like Desmond Clark or Howell himself had begun to adopt in Palaeolithic East African sites, based on the application and interpretation of an anthropological and multidisciplinary concepts. These interventions opened a methodological line that is still valid today. However, the campaigns carried out in Torralba and Ambrona had unfortunately very little Spanish participation, almost exclusively reduced in the archaeological aspects to Dolores Echaide. The first phase (1961-63) had virtually no impact on the development of the research on the Lower Palaeolithic in Spain; in a later stage (1980-1983), despite the work in Ambrona being partially funded and supported by Hispanic entities, the integration of Spanish archaeology into the research of Ambrona was also still poor.

\footnotetext{
(1) CENIEH. Paseo Sierra de Atapuerca n³, 09002 Burgos.

Correo-e: manuel.santonja@cenieh.es.

Id. ORCID: https://orcid.org/0000-0001-5848-9011
} 


\section{EL PROYECTO INICIAL DE F. C. HOWELL EN TORRALBA Y AMBRONA. INCORPORACIÓN DE DOLORES ECHAIDE AL EQUIPO DE INVES- TIGACIÓN}

Torralba y Ambrona constituyen desde hace tiempo referencias destacadas del Paleolítico antiguo europeo. A principios del s. XX las excavaciones realizadas por el Marqués de Cerralbo revelaron su magnitud, y en la década de 1960 Clark Howell renovó la atención internacional sobre ambas localidades. Se puso en marcha entonces un singular proyecto inspirado en el concepto antropológico, que investigadores como el propio Howell, o Desmond Clark, comenzaban a definir y aplicar en sus investigaciones en África oriental. Torralba y Ambrona fijaron en dos etapas el primer escenario europeo de aquella espectacular innovación. La primera y más destacada en 1961-1963 y la segunda de 1980 a 1983. La arqueóloga Dolores Echaide (19282018), recientemente fallecida, desempeñó en las dos un papel tan relevante como desconocido. El profesor norteamericano consiguió aglutinar un equipo interdisciplinar en el que colaboraban relevantes especialistas, a su lado jóvenes licenciados que en Torralba o en Ambrona establecían por primera vez contacto directo con los trabajos de campo. Echaide ocupó entre ellos un lugar singular y su aportación resultó clave, asumiendo tareas de responsabilidad cuyo conocimiento apenas ha trascendido más allá del círculo inmediato en torno a los proyectos, y no ha llegado a valorarse en su justa medida. Cuando se conoce en detalle es posible asegurar que sin su contribución las campañas en ambas localidades, en especial las de Ambrona, se hubieran visto muy mermadas. En esta revisión, que apoyados principalmente en la documentación elaborada por ella misma dedicamos a su memoria, pretendemos mostrar aspectos en buena medida inéditos y con indudable valor historiográfico, de las actuaciones arqueológicas en Torralba y Ambrona en las que Echaide participó. Incorporamos algunas referencias a su entorno familiar, formación y ejercicio profesional que pueden ayudar a comprender mejor sus aportaciones.

Dolores Echaide pertenecía a una cultivada familia donostiarra. Su padre, Ignacio María Echaide (18841962), ingeniero de profesión, fue presidente desde 1951 de Euskaltzaindia, la Real Academia de la Lengua Vasca. Ella estudió Peritaje mercantil y comenzó en Madrid en 1951 Filosofía y Letras, estudios que tuvo que interrumpir en 1953, con los dos primeros cursos comunes aprobados, para ocuparse de la casa familiar. Tarea en la que en 1959 tomó el relevo su hermana Ana, facilitando a Lola reanudar en la Universidad de Zaragoza los estudios iniciados en Madrid.

En julio de 1960 Howell visita por primera vez Torralba, motivado por la información recibida de Luis Pericot, contacto en el que desempeñó un papel clave y hasta ahora bastante ignorado el antropólogo norteamericano Sol Tax, padre de Susan Tax, esposa de
Leslie Freeman (Straus, 2016: 213-214). La evidente conservación de yacimiento y los restos superficiales le deciden a excavar, y organiza la primera intervención para el verano siguiente. Lola Echaide (LE en lo sucesivo) cursaba entonces en Zaragoza $4^{\circ}$ curso, hablaba inglés, francés y algo de alemán y disponía de otra titulación anterior. Esa formación sin duda pesó en la decisión de Antonio Beltrán -Arqueólogo Delegado del Ministerio de Educación Nacional de la zona de Aragón- para designarla como delegada española en la excavación de Torralba, donde acudió acompañada por Blanca Izquierdo y Maribel Bea, amigas y compañeras de estudios (Fig. 1).

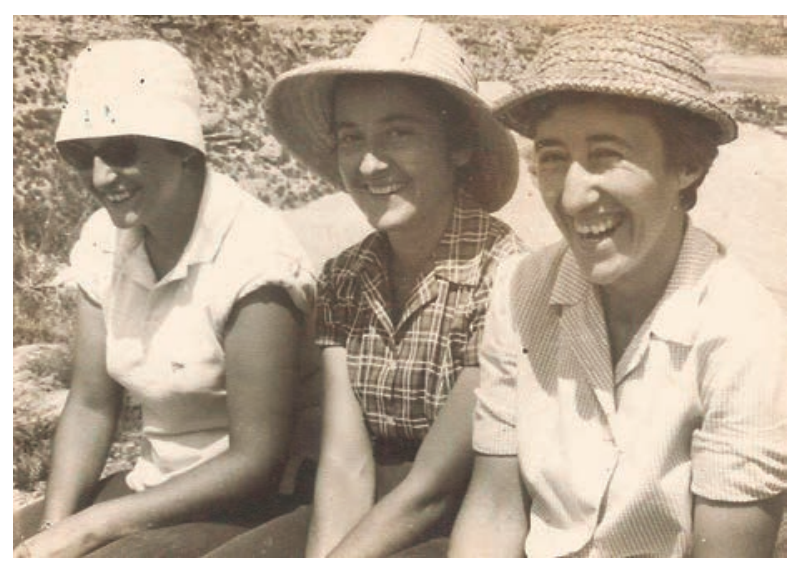

Fig. 1. Izq.-Dcha.: Blanca Izquierdo, Maribel Bea y Lola Echaide. Campaña de 1961 en Torralba (Archivo Echaide. CENIEH). / Left-right: Blanca Izquierdo, Maribel Bea and Lola Echaide. Torralba 1961 campaign (Echaide File, CENIEH).

El informe que LE remitió al Servicio Nacional de Excavaciones permite conocer de forma precisa la duración total de la campaña, del 7 de agosto al 20 de septiembre de 1961en Torralba y algunos días, entre el 17 y el 25 de agosto, en Ambrona, donde se realizó bajo el control directo de LE una primera cata exploratoria en la parte occidental del yacimiento. Además de participar activamente en todas las tareas, cometido que no correspondía ni se esperaba de una delegada ministerial, se ocupó de auxiliar a Howell en la organización del equipo de obreros reclutado en los pueblos inmediatos, y de actuar como traductora siempre que fue necesario. En especial se ocupó de las relaciones con instancias oficiales, desde la Guardia Civil a las autoridades locales, así como de traducir documentos e informes, entre ellos la crónica enviada por Howell al VII Congreso Nacional de Arqueología celebrado en Barcelona en el mismo mes de septiembre. La información que transmitió al Ministerio concreta la destacada participación de Desmond Collins y Peter Taylor, alumnos ambos de Howell que se empezaron a formar en Torralba en el trabajo de campo. Mencionaba también que K.W. Butzer realizó estudios geológicos del 13 al 29 de agosto y que $\mathrm{P}$. Biberson se incorporó por invitación de Howell en la última semana de actividad, pasando 
a formar parte del equipo de investigación. Finalmente recogía la colaboración esencial de José Viloria, enviado por el director del Museo Nacional de Ciencias Naturales, E. Hernández Pacheco, en el embalaje de los restos aparecidos, entregados en dicho centro y en el Arqueológico Nacional. Así mismo que E. Aguirre asumió la responsabilidad del estudio paleontológico y realizó una breve visita final a la excavación.

En la siguiente campaña, ya licenciada en Filosofía y Letras, LE quedó plenamente incorporada al equipo dirigido por Howell. El permiso de excavación emitido el 10 de julio de 1962 por la Dirección General de Bellas Artes siendo titular Gratiniano Nieto (Fig. 2), autorizaba al Dr. F. Clark Howell la realización de excavaciones en Ambrona, sin mencionar Torralba, donde también se excavó, "bajo la dirección de D. Francisco Jordá Cerdá como Delegado español; y con una misión constituida por los Srs. expresados al margen". Esa relación, con nombres a veces mal escritos, estaba integrada por "Srta. María Dolores Echaide, L.G. Treeman, D. Collins, Thomas Lynch, P. Biberson, Srta. J. Méndez Amor, Rvdo. P. A. Aguirre, Sr. Harl Butzer".

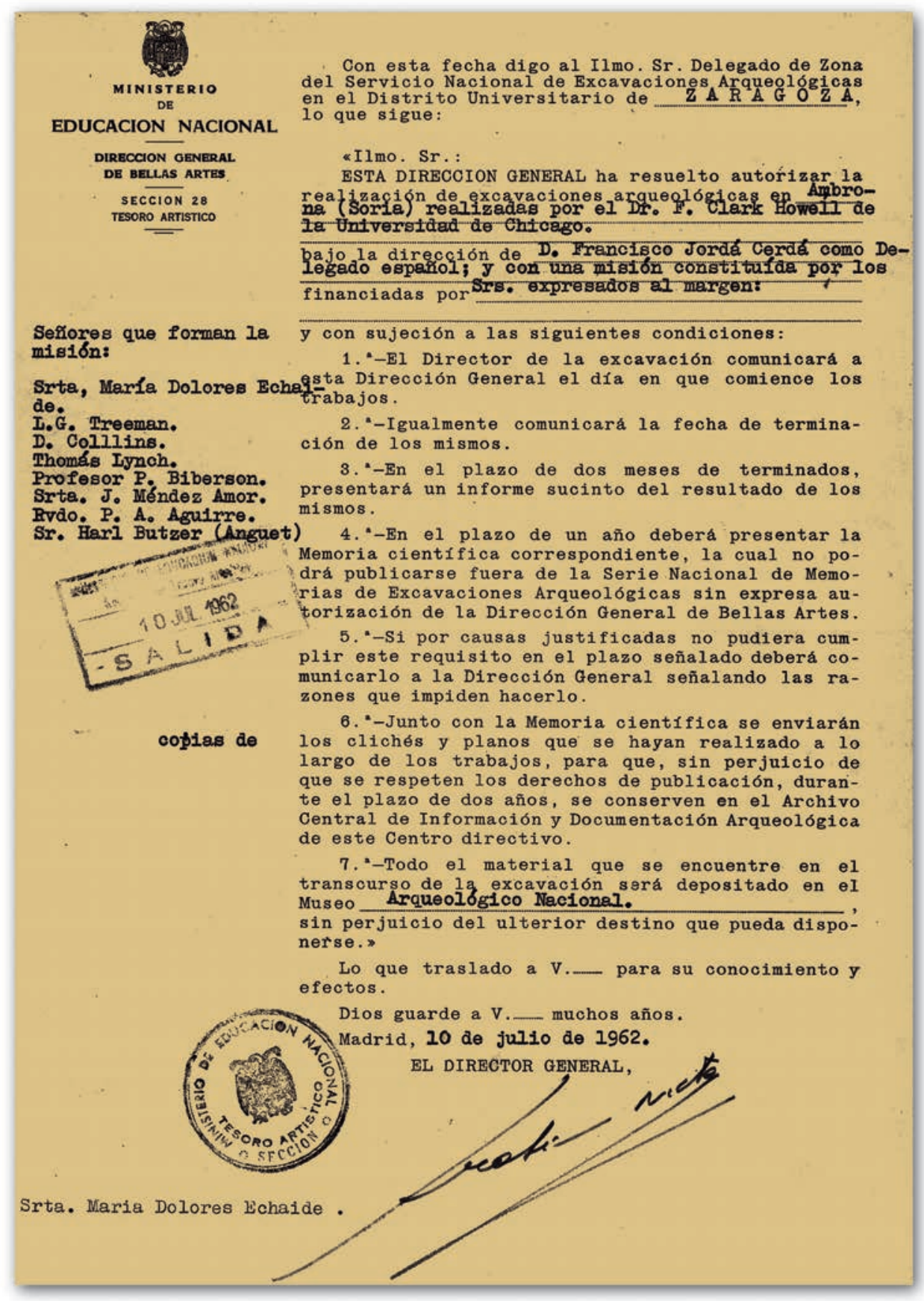

Fig. 2. Permiso de excavación para Ambrona concedido en 1962 a Clark Howell, no se menciona Torralba en el permiso. Dolores Echaide ocupa el primer lugar en el equipo de investigadores (Archivo Echaide. CENIEH). / Digging authorization for Ambrona granted in 1962 to Clark Howell, Torralba was not included. Dolores Echaide ranks first in the team of researchers (Echaide File, CENIEH). 
Además de las personas mencionadas en el permiso, también colaboraron en la campaña Susan Tax, Paul Mellars y Carmel Schrire. El nuevo delegado ministerial en la excavación, Francisco Jordá, se limitó a realizar breves visitas, sin participar directamente en las labores cotidianas ni aportar discípulos propios. Las labores transcurrieron entre el 2 de julio y el 9 de septiembre (Howell, 1965: 8) y durante seis semanas también se excavó en Torralba. LE se incorporó el 12 de agosto, centrando su actuación en Ambrona (Fig.3). Según recoge en sus notas, en ausencia de Howell la dirección recaía en $\mathrm{P}$. Biberson, auxiliado por Freeman y Lynch, que se ocupaban fundamentalmente del registro topográfico. Sin embargo Howell señala en un manuscrito inédito sobre las excavaciones de Ambrona que forma parte del conjunto documental de LE depositado en el CENIEH, del que más adelante nos ocuparemos, la particular posición mantenida por P. Biberson. Este investigador defendía que en los yacimientos paleolíticos al aire libre los momentos de ocupación tenían que ser singulares, criterio que le llevaba a rechazar que pudieran existir niveles de ocupación sucesivos tanto en Torralba como en Ambrona. Ese criterio determinó, según Howell, que Biberson acabara relegado a un papel secundario dentro del equipo.

Las actividades que describe LE coinciden con las recogidos en las publicaciones de los excavadores -p.e. Howell 1965-, detallando la apertura en Ambrona de tres trincheras, "Norte", "Inferior" y "Central", que respectivamente describe como "la más rica en fauna", solo con "pequeños fragmentos y bastante carbón" y "con restos limitados de huesos y útiles". Las relaciones con los obreros contratados en los pueblos limítrofes y la organización y control de sus labores fue una de las principales funciones que desempeñó, auxiliada por Blanca Izquierdo. Nos ha transmitido un fichero detallado de los 27 obreros empleados en el que incluye la edad, a veces la localidad, así como una valoración de las capacidades y habilidades de trabajo (Fig. 4). De los 15 en que consta la procedencia 7 venían de Horna, 5 de Ambrona y 1 de Miño, de Torralba y de Mojares. Uno de los de Ambrona era Pedro Puerta, que sería nombrado guarda del yacimiento en 1963, ya construido el primer museo, y que participó de manera destacada en todas las campañas de Howell, así como en la realizada en 1993 por Santonja y Pérez González en Ambrona, aportando indicaciones que fueron de gran utilidad. Estos trabajadores se distribuían en cuadrillas de 5 en cada uno de los cuadros de $3 \times 3 \mathrm{~m}$, siempre bajo la supervisión de un técnico responsable de levantar planos a medida que se avanzaba. Los visitantes que recibió la excavación dan buena idea del eco internacional que despertó desde el principio la intervención de Howell. Entre otros LE cita a R. J. Braidwood, Desmond Clark, Sonia Cole, James B. Griffin, T. Dale Stewart y Sol Tax.

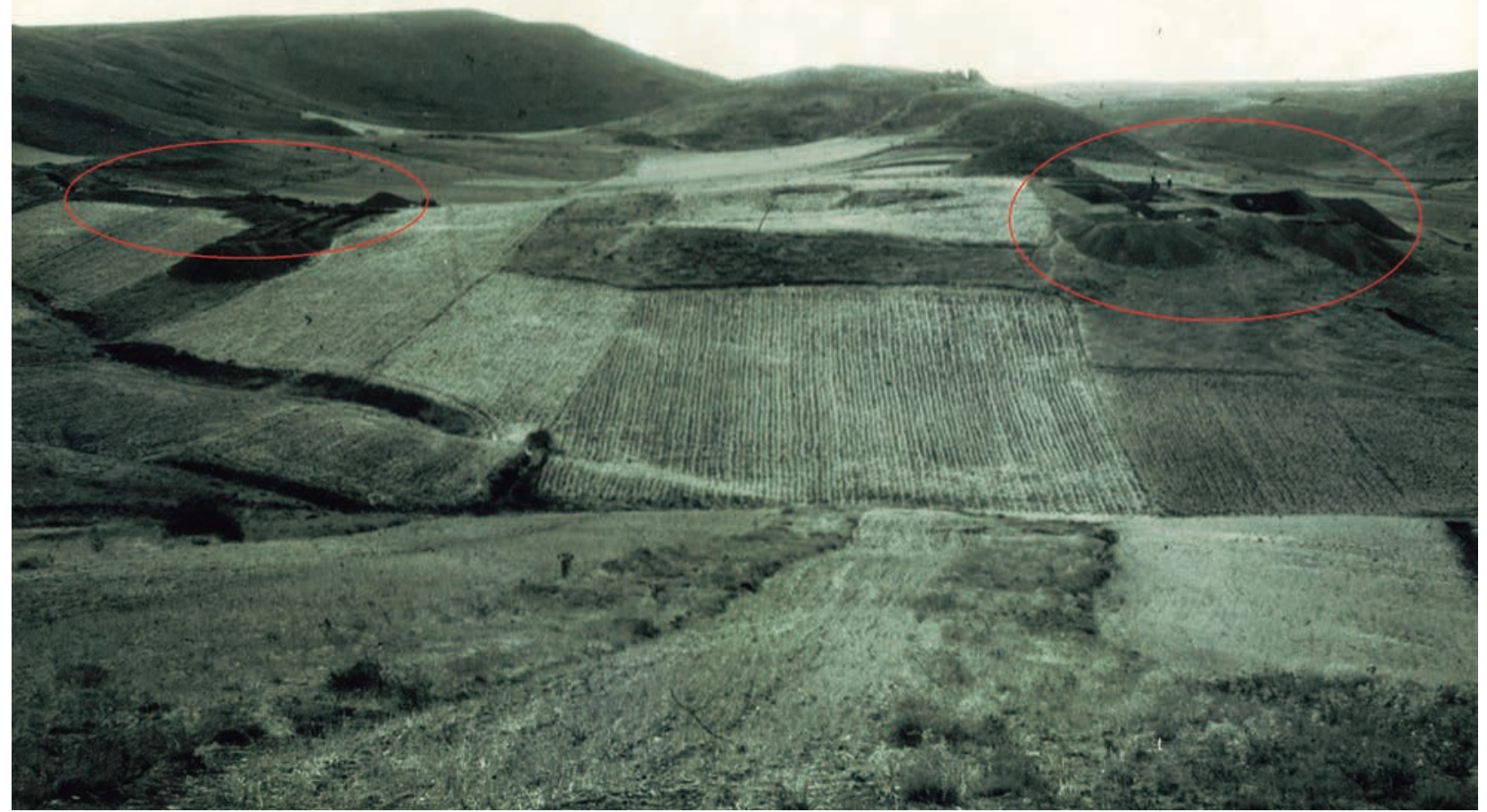

Fig. 3. Vista general de la excavación de Ambrona en 1962, antes de la construcción del primer museo. En el centro se aprecian huellas de las excavaciones realizadas por Cerralbo en 1914-1916 (Archivo Echaide. Cenieh). / Overall view of the excavation of Ambrona in 1962, before the building of the first museum. In the center you can see vestiges of the Cerralbo diggings in 1914-1916. (Echaide File. CENIEH). 


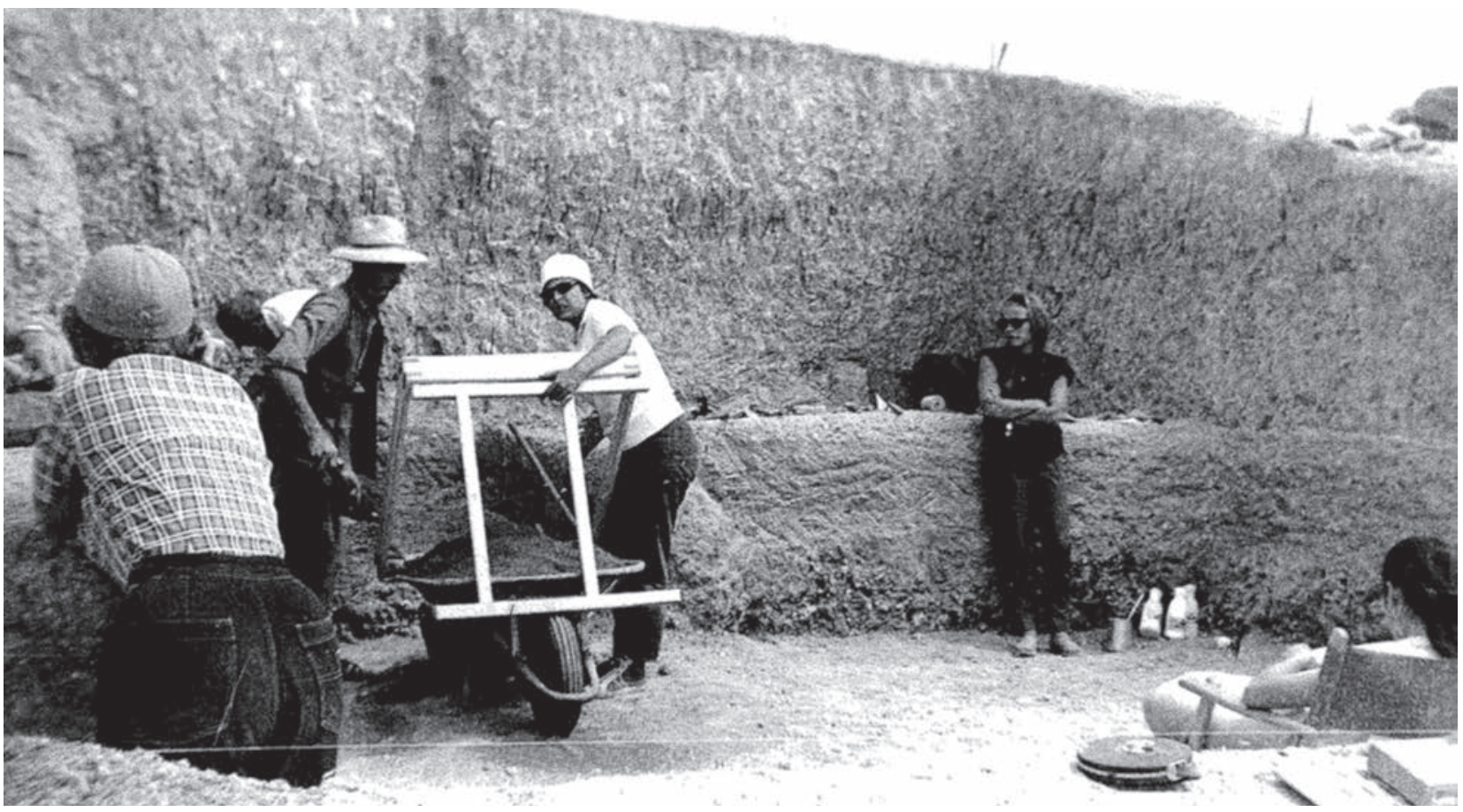

Fig. 4. Izq.-Dcha.: Dolores Echaide, Ezequiel Rupérez (obrero contratado, herrero de Horna, con 28 años de edad en la fecha. Echaide le describe en su cuaderno de campo como "Bueno cuando tiene compañeros trabajadores o cuando está de humor"). Blanca Izquierdo, Françoise Biberson y Maribel Bea. Ambrona, campaña de 1962 (Archivo Echaide. CENIEH). / Left-right: Dolores Echaide, Ezequiel Rupérez (28-year old contracted worker, blacksmith from Horna. Echaide describes him in her field notebook as "Good when he has fellow workers or when he is in the mood"), Blanca Izquierdo, Françoise Biberson and Maribel Bea. Ambrona 1962 campaign (Echaide File. CENIEH).

La larga campaña de 1963 aparece ampliamente descrita por LE en un pormenorizado diario que aporta detalles desconocidos hasta ahora. En Ambrona se excavó algo más de tres meses, desde el 27 de mayo hasta los primeros días de septiembre, y tanto en la
Loma de los Huesos -el emplazamiento clásico- como en la ladera situada enfrente, mencionada como "Camp North". La actividad en Torralba duró diez semanas a partir del 24 de junio (Fig. 5). El año anterior se había comprobado en Ambrona que la densidad de restos

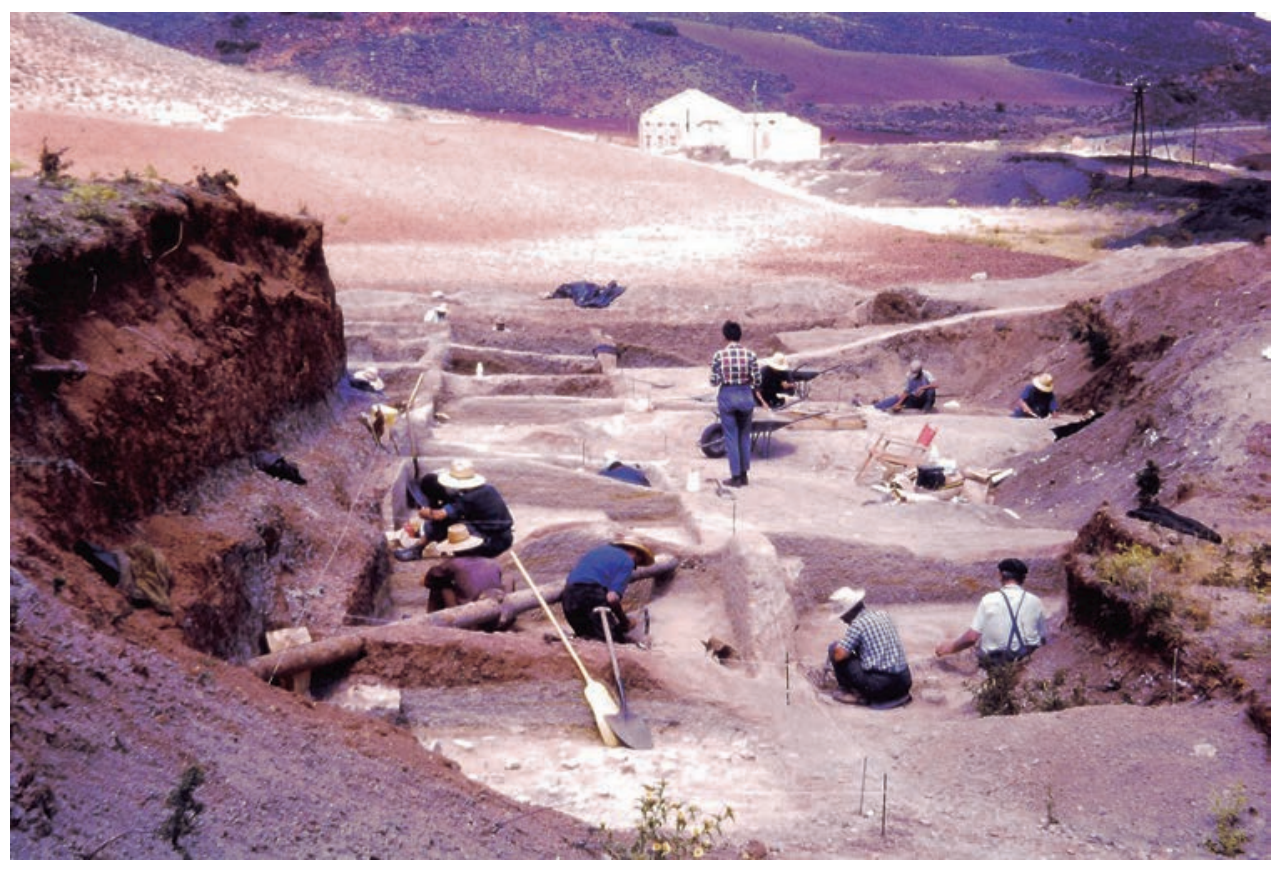

Fig. 5. Excavación de Torralba campaña de 1963. Se observa la tubería que llevaba agua a la estación de ferrocarril, cuya instalación puso al descubierto en 1888 los primeros restos de elefante identificados en el yacimiento. En el centro, de pie y de espaldas, Dolores Echaide (Archivo Echaide. CENIEH). / Digging Torralba, 1963 campaign. The installation of the water pipe to the railway station uncovered in 1888 the first remains of elephants identified in the site. Dolores Echaide standing in the foreground, (Echaide File, CENIEH). 
era mayor en el área central. En consecuencia en 1963 se abandonó el sector occidental, donde no se volvería a intervenir, y se trasladó el interés a la zona centro, tanto al oeste de la sección abierta en 1962 -Área B, 38 cuadros, $342 \mathrm{~m}^{2}$ - como al este -Áreas 1-4, 52 cuadros, $468 \mathrm{~m}^{2}$-, excavándose en total más de $800 \mathrm{~m}^{2}$. El 3 de junio, fecha en que se incorporó LE, se trabajaba simultáneamente en Ambrona en los cuadros 38B, 39A, 39B, 39C, 39E, 40D y 40E, tres obreros y un supervisor en cada uno. En Torralba los trabajos se realizaban bajo el control directo de Freeman, auxiliado por Blanca Izquierdo, mientras LE continuaba en Ambrona con Howell, "Mike" -Michel Moseley-, "Richard" -Richard Klein-, "Craig" -Craig Morris- y 17 obreros hasta el 9 de julio, fecha en que se traslada a Torralba, reincorporándose a Ambrona de nuevo el 28 de ese mismo mes. A partir del 19 de agosto las operaciones en Ambrona se centrarían en el área del futuro museo, denominada STE -Slit Trench Extension-, ocupándose LE con Richard y Mike del control y registro. Describe el sedimento como "grey marl" y "gritty marl" antes de alcanzar el pavimento, en el que reconocían al menos dos capas, aportando esquemas de la estratigrafía y observaciones sobre densidad de restos.

En su diario LE recoge con frecuencia apreciaciones personales acerca del desarrollo de la excavación y de la interpretación estratigráfica (Fig 6). El 3 de julio anota que "el pavimento sigue inclinándose. No me gusta cómo vamos cavando -sic-, lo hacemos de parte alta del pavimento hacia abajo, se hacen enormes paredes, no seguimos cuadros...". Pocos días después, el 8, opinaba de manera contundente que "no hay quien entienda la estratigrafía". Otra observación del 30 de julio sugiere la existencia en el espacio central de Ambrona de lo que actualmente se interpreta como "unidad estratigráfica media" con Paleolítico medio (Pérez González et al. 2005): "la parte inferior de la Ph IV, un "gray grit", es muy rica en dientes de caballo y lascas... a mi parecer los raspadores y lascas son de una técnica más avanzada, casi sobre hojas". El 16 de agosto, después de las revisiones estratigráficas de Butzer, señala que está "acabada el área del 49 y $50 . . . "$ y que de acuerdo con las observaciones de Butzer tienen mal la estratigrafía, la correcta era Ph IV con varios "red grit" y al fondo una "gray grit", luego "Ph III marga gris, gray grit, brownish grit, gravel y sandy grit y (a veces) B gravel... luego Keuper... Solamente en 46 F y $\mathrm{G}$ y en $47 \mathrm{~F}$ y $\mathrm{G}$ se encuentra la Ph I". En esta secuencia parece reconocerse a techo con claridad la actual "Unidad estratigráfica media".

Echaide sigue recogiendo de manera minuciosa la composición del grupo y los visitantes recibidos a lo largo de la campaña. Constan los nombres de 19 obreros, la mitad nuevos pero muchos, Pedro Puerta por ejemplo, bastante experimentados. Se reciben visitas de profesores muy conocidos, el antropólogo Sol Tax de nuevo, Arthur J. Jelinek, R. Nougier, ApSimon, R. Paepe, L. Pericot, J. González Echegaray, E. Ripoll y Luis

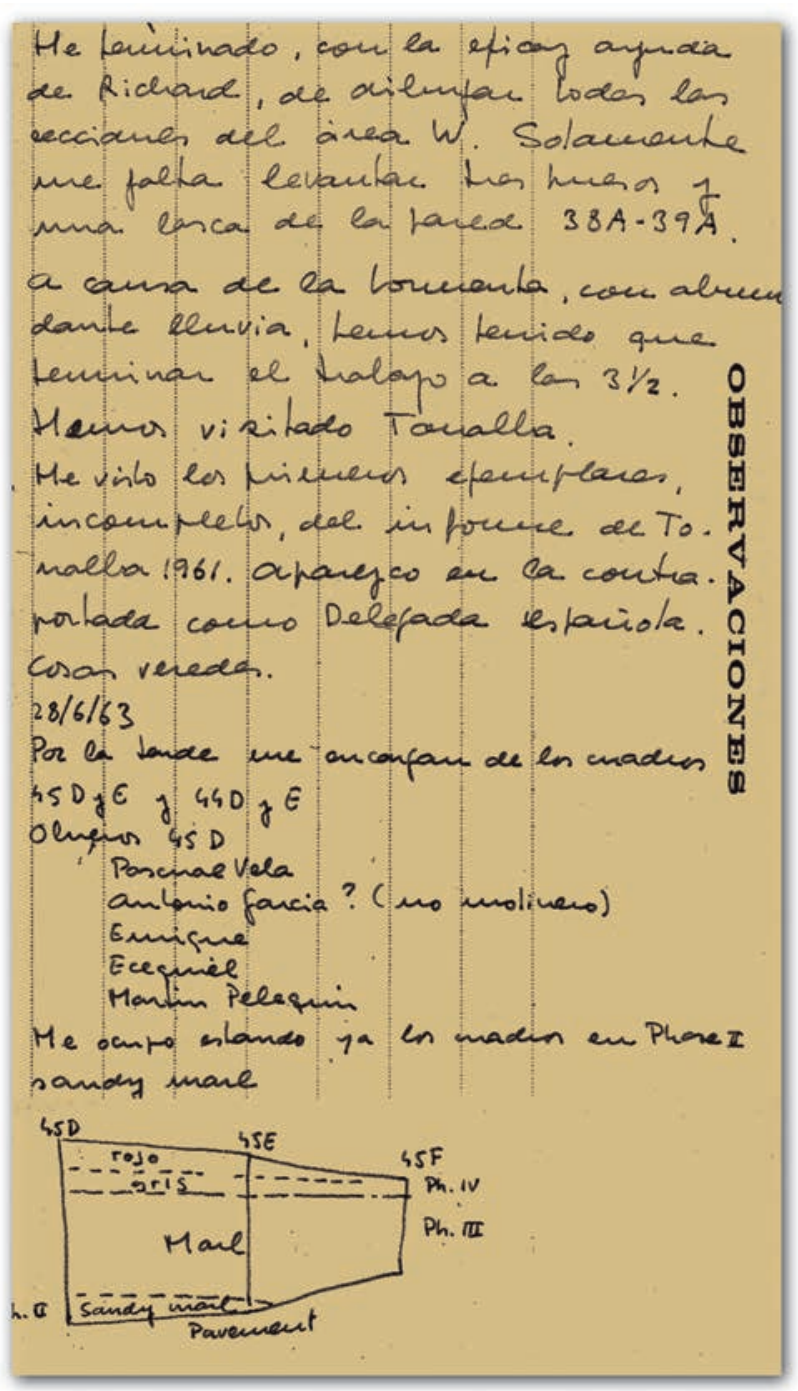

Fig. 6. Página del cuaderno de campo de Dolores Echaide. Excavación de Ambrona, 27 y 28 de junio de 1963 (Archivo Echaide. CENIEH). / Page of the Dolores Echaide's field notebook. Digging Torralba, 1963 campaign. June 27 and 28 (Echaide File, CENIEH).

Pericot. Destaca la del matrimonio Bordes, François y Denise, que llegaron el 25 de junio permaneciendo varios días en Ambrona. Menciona así mismo que bajo la dirección de Clark Howell, ausente entre los días 13 y 26 de agosto, colaboraron en los controles técnicos Biberson, Freeman, Michel Moseley -17 de junio al 23 de agosto-, Thomas y Barbara Lynch -hasta el 22 de junio-, Craig Morris, Richard Klein, Karl Butzer -13 al 17 de agosto-, Peter Taylor, Blanca Izquierdo, Maribel Bea y ella misma. Emiliano Aguirre estuvo en la excavación el 20 de junio, acompañado de José Viloria, y la financiación de los trabajos continuó siendo asumida por The National Science Foundation.

En 1963 no se interrumpió totalmente como habitualmente se cree la actividad en Ambrona. En la campaña de ese año se tomó la decisión de levantar una construcción que permitiera conservar alguna de las 
áreas de la parte central rica en restos de elefantes. El diseño del edificio, muy sencillo, lo realizó LE con E. Aguirre, quien consiguió financiación del Ministerio de Información y Turismo y de la Diputación de Soria. La obra estaba finalizada el 15 de noviembre de 1963. Poco después el Ministerio de Educación Nacional aceptó hacerse cargo del museo, designando a Pedro Puerta como guarda (Aguirre 2005). Para rematar la operación fue preciso consolidar los restos faunísticos y alguna otra labor complementaria. Estas tareas, que han sido descritas exclusivamente por LE, fueron dirigidas en 1964 por Aguirre y ejecutadas bajo el control directo de LE. Entre el 15 y el 30 de septiembre y con la participación de Blanca Izquierdo y Maribel Bea -las compañeras de los años universitarios en Zaragoza-, junto a Pedro Puerta y algunos obreros contratados en la comarca como J.A. Riosalido y Jesús Navalpotro, se profundizó hasta el Keuper en algún punto -"Keuper removido de aspecto arenoso con mezcla de margas y líneas calcáreas"-, se tomaron muestras para sedimentología y se limpió la superficie del pavimento en todo el museo. Aguirre no intervino de manera directa en los trabajos, ocupándose fundamentalmente de aportar carpinteros y personal técnico para completar la consolidación de los restos faunísticos.

\section{EL PARÉNTESIS DE 1965-1980 INTERRUMPIDO EN 1973}

Una vez finalizada la instalación del museo, la intención de Howell era reanudar la excavación. Pero a finales de 1965 surgió una magnífica oportunidad en la cuenca del río Omo, y el profesor norteamericano, sin renunciar a volver a Soria, decidió asumir la organización de este irrechazable proyecto. Las campañas en Etiopía se prolongaron hasta 1974 y varios años mas los estudios derivados, imponiendo un largo paréntesis en la investigación de los yacimientos sorianos.

La intensa actividad desplegada en Torralba y Ambrona había aportado a LE una experiencia del máximo nivel. Una importante formación que procuró consolidar y que no interrumpió en lo que se perfilaba como una tregua del plan iniciado en 1961. En la primavera de 1963, del 16 de abril al 4 de mayo, formó parte del equipo de excavación de Aitzpitarte IV (Rentería, Guipúzcoa), con J. M. Barandiarán; campaña en la que también participaron Blanca Izquierdo y Maribel Bea, que aparecen con LE como colaboradoras en el informe anual de estos trabajos -cf. Munibe 16, 1-2: 12-23-.

El descubrimiento de un nuevo sitio achelense en Galicia, Gándaras de Budiño (Pontevedra), de cuya investigación se hizo cargo E. Aguirre (Aguirre 1964), le llevó allí en septiembre, pocos días después de terminar en Ambrona, aceptando ocuparse de la industria lítica. Este estudio fue concebido como núcleo de una tesis doctoral que no llegó a concluir, proyecto para el que en años sucesivos dibujó gran parte de las piezas obtenidas en la excavación de 1963 (Fig. 7). En todo caso el análisis que efectuó desembocó en la publicación de un notable artículo (Echaide 1971), que sigue siendo valorado y que constituía en ese momento el primer estudio
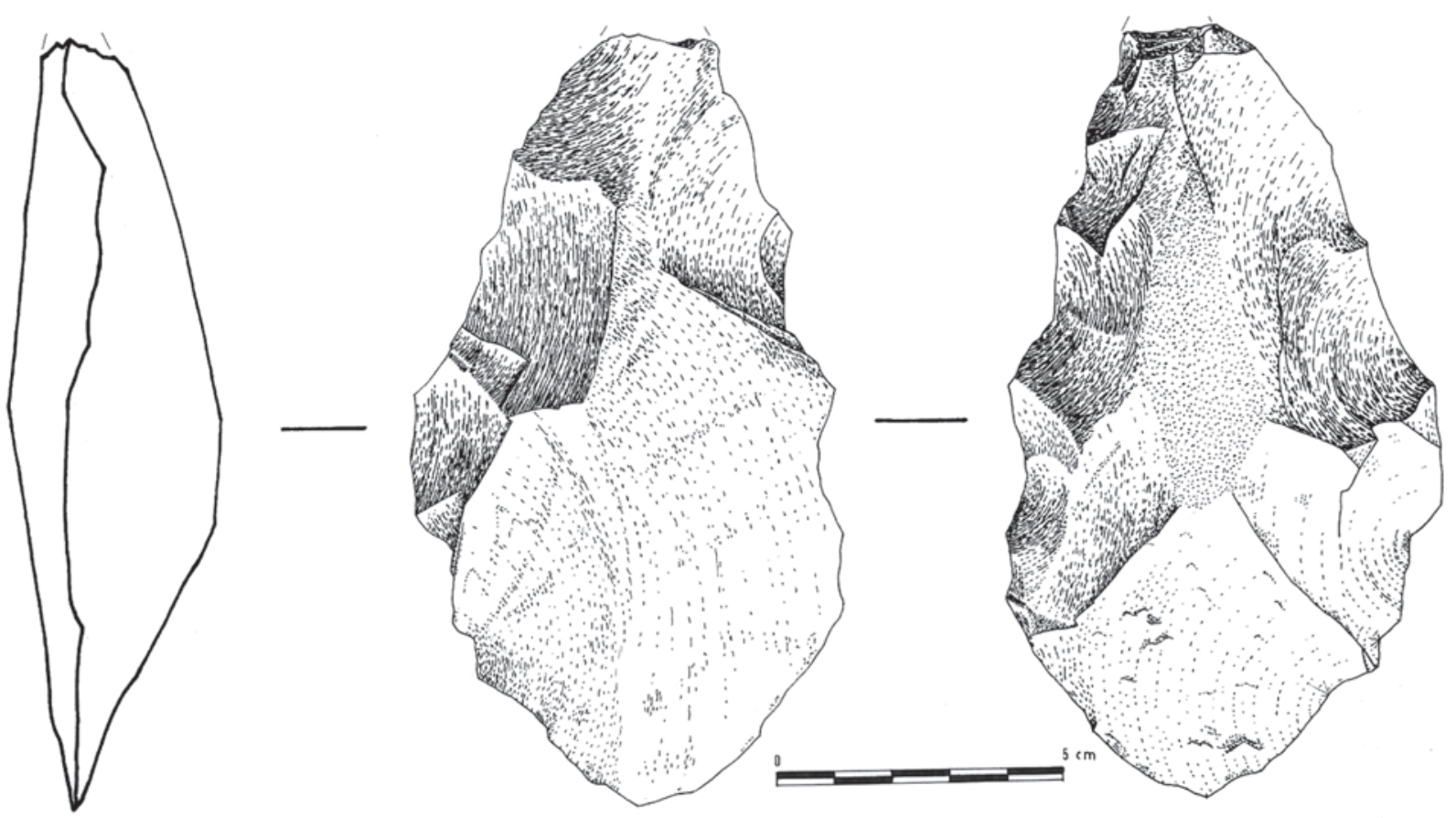

Fig. 7. Bifaz de la excavación de Gándaras de Budiño. Se trata de uno de los muchos dibujos inéditos de industria lítica de este yacimiento realizados por Echaide para su proyectada tesis doctoral. Referencia: dib. n 44, Bu 63, Cn p-b, 423, n⿳838 (Archivo Echaide. CENIEH). / Handaxe from Gándaras de Budiño. This is one of the many unpublished drawings of lithic industry of this site made by Echaide for her PhD. Reference: dib. $n^{\circ} 44, \mathrm{Bu} 63, \mathrm{Cn} p-b, 423, n^{\circ} 838$ (Echaide File, CENIEH). 
con metodología moderna sobre industria lítica del Paleolítico inferior de la Península Ibérica. Meses después, el 12 de octubre de 1963, LE viaja a Chicago con contrato de aquella universidad para estudiar con Howell la industria de Torralba y de Ambrona y pasar a limpio los planos, una labor que resultó fundamental para corregir errores de campo. Allí participaría con el resto del equipo en un congreso en San Francisco, presentando resultados de las excavaciones. Su estancia se prolongó hasta el 9 de mayo de 1964, fecha a partir de la cual vuelve a España y se ocupa en el MNCN de Madrid de organizar la fauna obtenida tanto en Torralba como en Ambrona.

La dedicación arqueológica de LE no se detuvo. Después de las operaciones de septiembre en el museo de Ambrona, en el mes de diciembre de 1964 recorre las graveras del Tajo desde Toledo a Portugal, conociendo de manera directa yacimientos entonces en pleno proceso de investigación, en especial Pinedo (Toledo). Realiza también un primer viaje a Marruecos en compañía de Biberson, entrando en contacto con los puntos clave de la secuencia paleolítica del Marruecos atlántico. En 1965 visita los principales enclaves paleolíticos de Aquitania, y excava en Lezetxiki con J. M. Barandiarán del 23 de abril al 15 de mayo. En 1966 efectúa una estancia en el I.P.H. de Paris con Pierre Biberson que se prolonga hasta marzo del 67, aprovechando para tomar un curso de dibujo de industria lítica con Michel Dauvois, el más reputado especialista que existía en la época (Fig. 8). En mayo vuelve a visitar al

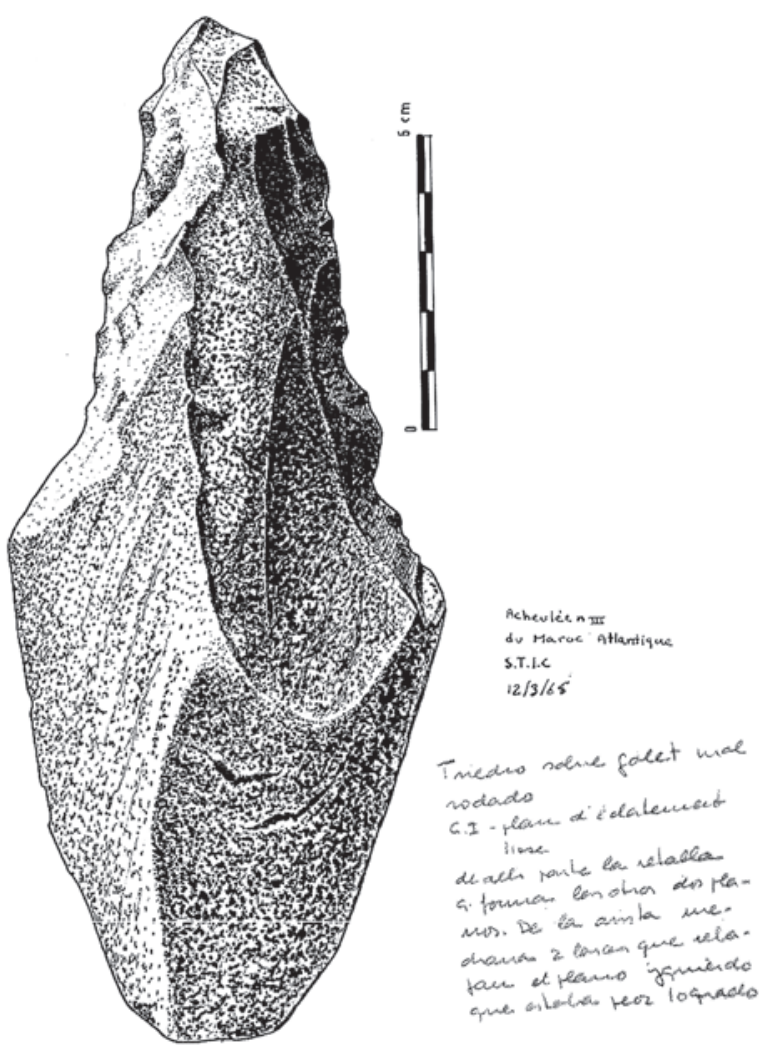

lado de Biberson los yacimientos de Casablanca y más tarde, en 1968, excava en Cueva Morín con Freeman y ya en 1969 con Jacques Tixier en La Faurelie II (Francia) y en Ksar'Aquil (Líbano), intervenciones todas ellas de un alto nivel metodológico que sin duda aportaron a LE conocimientos que aplicaría en Ambrona.

El retorno momentáneo a Ambrona en 1973 partió de Martín Almagro, a la sazón titular de la Comisaría General de Excavaciones Arqueológicas, decidido a ampliar la instalación museística de 1963 y añadir nuevos espacios aptos para albergar sala de exposiciones, almacén y dependencia para el guarda. Aunque el cumplimiento de la iniciativa se demoraría varios años, en 1973 se acordó excavar una zona inmediata al edificio existente con objeto de mejorar sus condiciones ambientales y facilitar la obra futura. Con la conformidad de Howell, que seguía dedicado en exclusiva al proyecto en Etiopía, la dirección de la excavación se encomendó a Emiliano Aguirre. El peso organizativo correspondió no obstante a LE, que en el diario que preparó empieza refiriéndose a la "inesperada" visita de Aguirre, recibida el 2 de junio en el domicilio donostiarra que compartía con Blanca Izquierdo. Aguirre les planteó la necesidad de excavar antes de construir el nuevo museo y LE supuso, y así lo anotó, que el requerir la participación de ellas sería más bien sugerencia de Howell. El plan de Aguirre era empezar el 10 o 12 de julio y continuar hasta finales de agosto, con siete ayudantes, dos o tres geólogos, otros tantos paleontólogos y "una arqueóloga", más Blanca y LE. A ese cuerpo técnico sumaba un fotógrafo, un restaurador, seis obreros y el concurso de una máquina excavadora. LE consideraba que Aguirre quería excavar demasiado, pero acabó -como Blanca- aceptando, motivadas por el presumible interés de Howell. Este visita Madrid y se reúne con Aguirre y LE el 16 de junio en el MNCN. En el encuentro se decide de manera precisa el sector a excavar y se encomienda a LE la subdirección arqueológica, compartiendo responsabilidad con otra subdirectora geóloga propuesta por Aguirre, Margarita Díaz Molina.

La campaña dio comienzo un mes después y disponemos de la información recogida en el diario de LE, que incorpora además fragmentos de otra crónica elaborada por Blanca Izquierdo. El 16 de julio Ilega LE

Fig. 8. Dibujo de un bifaz de Carrière S.T.I.C., yacimiento achelense próximo a Casablanca (Marruecos), realizado por Echaide probablemente durante la estancia realizada en 1966 en el I.P.H. de Paris, siguiendo el magisterio de Dauvois. Leyenda explicativa -en español y francés- de la mano de Echaide: "Triedro sobre galet mal rodado. C.I. (cara inferior) - plan d'eclatement lisse de allí parte la retalla q(ue) forma los otros dos planos. De la arista mediana (parten) 2 lascas que rebajan el plano izquierdo que estaba peor logrado" (Archivo Echaide. CENIEH). / Drawing of a handaxe from Carrière S.T.I.C., Acheulean site near Casablanca (Morocco), probably made by Echaide during her 1966 stay in the I.P.H., Paris, following the Dauvois teaching. Explanatory caption - in Spanish and French - by Echaide. (Echaide File. CENIEH). 
a Medinaceli y encuentra a Aguirre con Margarita Díaz y la restauradora Paloma Gutiérrez del Solar. Al día siguiente Aguirre, antes de empezar en Ambrona, decidiría ocuparse de unos restos de elefante aparecidos en la localidad de Mojares, a 14 km en dirección Sigüenza, en el Alto Henares. La excavación en Mojares se efectuó paralelamente a la de Ambrona en los siguientes días, pero los restos encontrados, una vez limpios y preparados para su extracción, fueron expoliados y no se conservan (Aguirre 2005: 50-52). De acuerdo con el diario de LE tan lamentable pérdida se produjo el 2 de agosto.

La organización del equipo de operarios para Ambrona y la delimitación de las superficies a excavar al sur del Museo se llevaron a cabo conforme al plan previsto y el control de Echaide. El 19 de julio terminarían LE y Blanca Izquierdo de preparar la zona, cuadriculándola al día siguiente y acomodando así 18 cuadrículas de $9 \mathrm{~m}^{2}$-una superficie de $162 \mathrm{~m}^{2}$-, de las que aporta croquis de situación. Relata cómo surge un problema con Aguirre para establecer el "punto 0" del sistema de registro, que finalmente se fija en la pared sur del Museo, donde aún permanece visible y operativo, si bien Echaide tiene que aceptar la imposición de otro plano 0 marcado en una de las estacas señalizadoras de las cuadrículas -evidentemente desaparecido-, precisando que se encontraba $38 \mathrm{~cm}$ por debajo del fijado en la pared del museo.

Los trabajos fueron avanzando con el propósito de alcanzar el pavimento, el nivel inferior de Ambrona, en toda el área. LE aporta información diaria de lo excavado y por quién, con comentarios que denotan alguna disconformidad organizativa. Por ejemplo el 27 escribe: "los jefes parecíamos Cabrera y yo", "la excavación, dadas las circunstancias y los excavadores no es tan absolutamente desastrosa como podría ser"; el 28 "todo normalmente anormal"; el 9 de agosto "pequeño lío por el hallazgo de un fragmento de lámina en C9"; el 10 "Todo igual, igualmente científico" y el 13 de ese mes "...Naturalmente muy pocos hallazgos". El 21 de agosto anota una descripción del estado de cada cuadro, unos en Keuper y ya terminados y otros sin empezar.

El 31 de agosto concluyó la campaña, efectuando después envíos de documentación a Clark Howell y también a Aguirre, que el 21 de enero del siguiente año se acerca de nuevo a San Sebastián, con objeto de recoger diapositivas y originales de planos y secciones y abonar los servicios prestados. Margarita Díaz Molina y Paloma Gutiérrez del Solar cumplieron su cometido como responsables de la geología y la restauración. De acuerdo con las anotaciones de LE intervinieron también otros especialistas: María Angélica Borrello registrada como "arqueóloga argentina"-, Juan Laguna -"restaurador"-, Antonio Ruiz Bustos -"geólogo y biólo- go"-, Enrique Soto y José Luis Sanz -"paleontólogos"- y Blanca y LE, que se auto identifican en el diario como "ex prehistoriadoras". Además una decena de estudiantes de diversas especialidades y 10 obreros contratados en los pueblos próximos, casi todos ellos veteranos. Aparte de Almagro, Pierre Biberson, que ya no participaba, visitó la excavación en varias ocasiones.

Después de 1973 la labor en Ambrona se interrumpió de nuevo varios años, aunque no la relación de LE con la comarca. En 1974 compra un terreno en el pueblo y en el verano construye una casa en la que primero pasará los veranos y luego temporadas más largas, para finalmente en 1989 establecer en ella, con Blanca Izquierdo, su residencia habitual. La relación con Howell no cesó, y tampoco con Freeman, a quien visitó en la excavación de Abric Agut (Capellades, Tarragona) en 1976.

\section{SEGUNDA ETAPA EN AMBRONA (1980-1983)}

En 1980 comenzó un nuevo ciclo en Ambrona -no se volvió a excavar en Torralba- con participación plena de LE. En el permiso emitido por la Dirección General del Patrimonio Artístico, Archivos y Museos siendo director general Javier Tussell y Subdirector de Arqueología Manuel Fernández Miranda, junto a HoweII y Freeman figura también como co-director Martín Almagro Basch, que aparece además en primer lugar. El Instituto Español de Prehistoria sería la institución patrocinadora, contando el proyecto con subvenciones de la National Science Foundation -subvención a Freeman y Howell-, el Comité Conjunto Hispano-Norteamericano para Asuntos Educativos y Culturales -ayuda concedida a M. Almagro- y donativos de la L.S.B. Leakey Foundation. Los preparativos de la campaña comenzaron a mediados de junio, concertando acuerdos con Celestino y Tomás Navalpotro dueños de las parcelas en que se iba a excavar. A la vez José Luis Argente, director del Museo Numantino, preparó por encargo de la Dirección General de Patrimonio la documentación necesaria para realizar las expropiaciones imprescindibles para construir las nuevas dependencias, contemplando también la posibilidad de adquirir la totalidad del yacimiento. Como en etapas anteriores otro asunto que asume LE en los primeros días es la contratación de obreros y el salario a pagarles, gestión que demostraba la confianza que seguía depositando en ella Howell (Fig. 9).

La excavación arrancaría el 17 de junio con la limpieza de la zona al oeste del Museo de 1963. Se arrasa con pala mecánica una amplia plataforma (Fig. 10) eliminando depósitos que se consideraban estériles, lo que ha originado graves problemas para la interpretación estratigráfica del yacimiento, al eliminar la continuidad de los niveles entre los sectores Este y Oeste (Pérez González et al. 2005: 185). El 26 se acabó de 


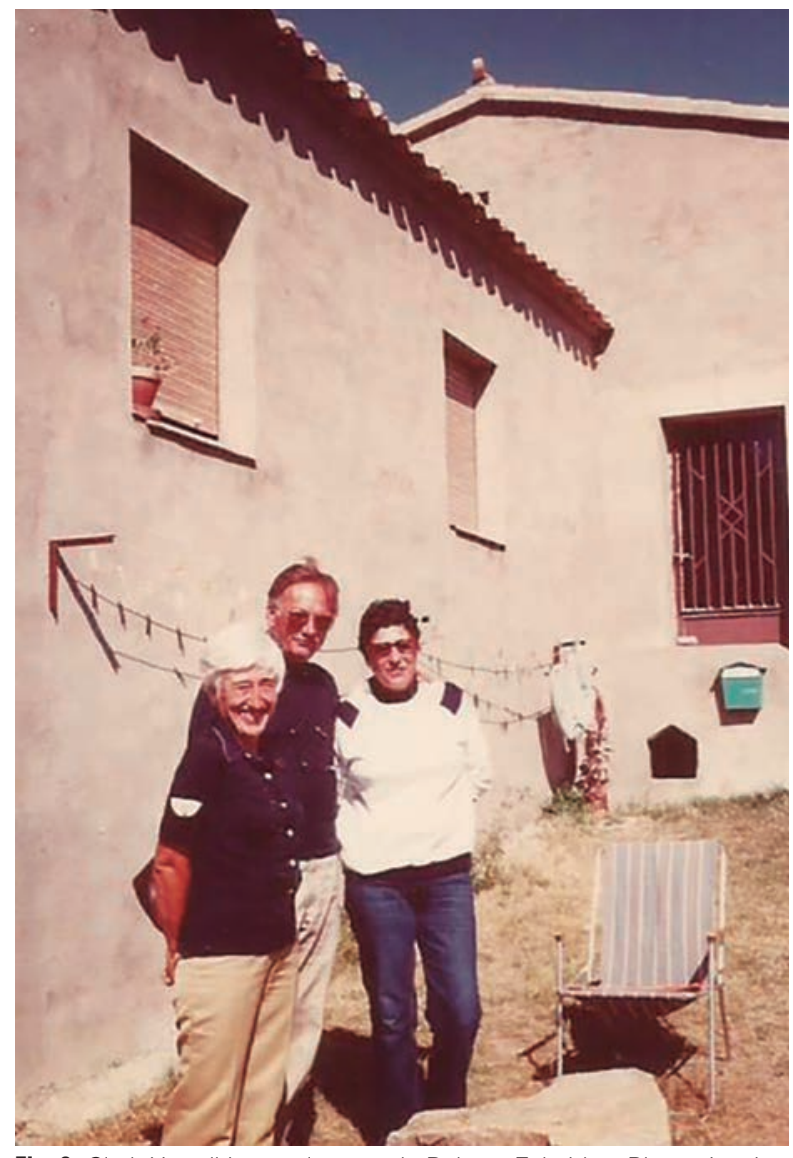

Fig. 9. Clark Howell junto a la casa de Dolores Echaide y Blanca Izquierdo. Ambrona 1980 (Archivo Echaide. CENIEH). / Clark Howell next to the Dolores Echaide and Blanca Izquierdo house. Ambrona 1980 (Echaide File, CENIEH).

rebajar toda la zona, aunque la pala siguió rebajando por el NE del museo, limpiando cortes "monte arriba" "en las "tierras rojas" del área abierta en 1963 en el este del yacimiento, donde el día 27 se profundiza un pozo de control que alcanza el Keuper. En agosto se abrirían nuevos cuadros por el extremo N "buscando la orilla del lago". Durante todo el tiempo hay distintas opiniones acerca de cómo conectar el sistema de cuadrículas de los años 1960 con el nuevo, y también se plantean problemas con las interpretaciones estratigráficas, poco claras para los excavadores.

Junto a LE, que intervino como "supervisora", la excavación contó en el mes de agosto con la contribución destacada de Charles Turner -8 al 22-, de K.W. Butzer -11 al 25- y de H. P. Schwarcz en la segunda quincena. Fue además la que tuvo mayor participación de personal técnico español, en concreto Gerardo Vega Toscano, Inmaculada Rus, Sergio Ripoll y Carmen Cacho, enviados por Almagro. Otros ayudantes fueron Frank S. Harrold -Universidad de Texas-, Roderic V. Moore -Universidad de California-, Alan Sokoloff -Universidad Hebrea de Jerusalen-, Carmen Sesé -MNCN, Madrid-, ocupada en la obtención de muestras para microfauna, y Judith Odgen, dibujante. Asimismo se contó con catorce obreros, y Juan Laguna, del MNCN, solucionó de nuevo la recogida y embalaje de la fauna. Colaboraron también excepcionalmente ese año numerosos estudiantes españoles (Lourdes Ortega, Charo Alonso, Ester Martín y José Sánchez Sastre -Valladolid-; Emilio Ruiz -Soria- Francesc Burjachs, Juan Oller, José Manuel Rueda y María Teresa Ros -Barcelona-, Javier Cochí y Enrique Gil -Zaragoza-, Octavio Collado -Teruel-, Enrique Gómez -Santander-, Ana María Martínez, Francisco Wandosell y Marco de la Rasilla-Madrid-) y algunos americanos (Brian D. Howell y Claudia McMahon, de California) e ingleses (William MacCormack, de Cambridge). No había precedente de una colaboración española tan nutrida, pero lamentablemente, aparentemente por decisión de Howell, no volvería a repetirse. El comentario de Howell hacia el final de la campaña, fue elocuente: "Clark me dice que el año que viene más obreros y menos estudiantes". El día 11 de septiembre se dio por terminada la temporada.

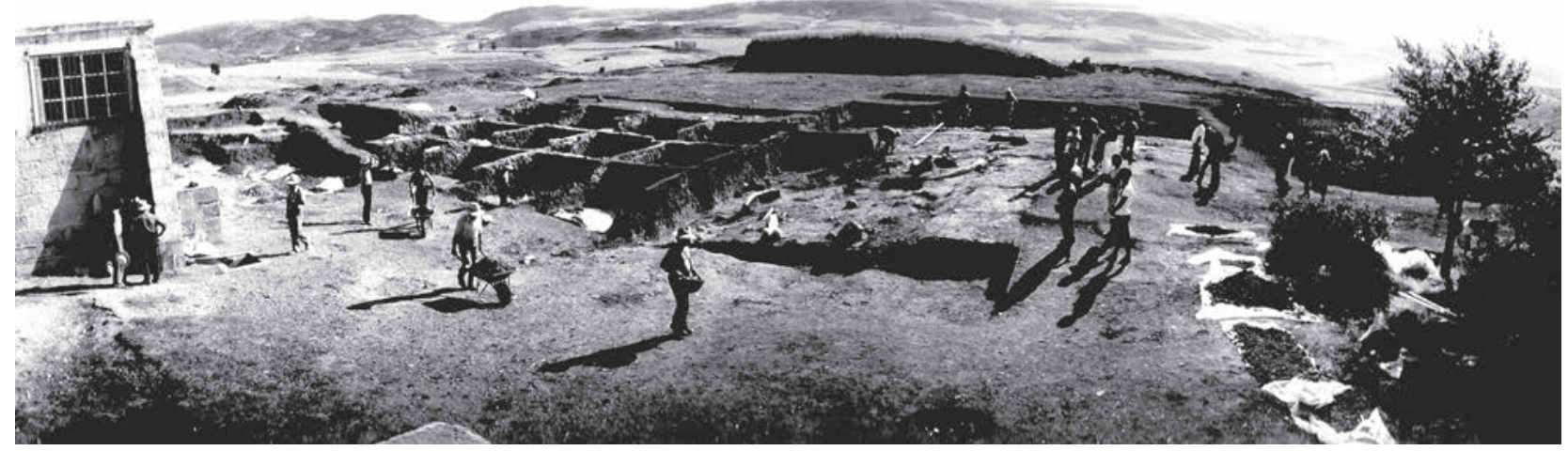

Fig. 10. Vista general de la gran superficie denominada Área 6, con una extensión de $369 \mathrm{~m}^{2}$, excavada en Ambrona al oeste del museo -cuya esquina NW aparece a la izquierda de la imagen- en la campaña de 1980. En la imagen se aprecia explanado, rebajado con pala mecánica, todo el sector oeste del yacimiento (Archivo Echaide. CENIEH). / General view of the large platform named Area 6 (369 $\mathrm{m}^{2}$ ) excavated in Ambrona to the west of the museum -whose NW corner can be seen on the left of the image- during the 1980 campaign. The western sector of the site levelled and lowered with mechanical means can be observed in the picture (Echaide File, CENIEH). 
La reanudación de los trabajos atrajo en 1980 notables visitantes. En algún caso -José Luis Argente, Gordon y Carla Ghetty y representantes de la Fundación Leakey- personas relacionadas con la gestión del proyecto, si bien la mayoría especialistas interesados en conocer los resultados obtenidos. Entre ellos se cita a Arthur J. Jelinek -Universidad de California-, Naama Goren -Universidad de Jerusalen-, Joaquín González Echegaray -Director de Altamira en ese momento-, V. Cabrera y F. Bernaldo de Quirós -Universidad Complutense-, Eduardo Ripoll -Universidad de Barcelona-, Emiliano Aguirre-MNCN, Madrid-, J. de Heinzelin -geólogo, Universidad de Bruselas-, Pilar López -CSIC- o Manolo Hoyos -MNCN, Madrid- en repetidas ocasiones, renunciando este finalmente a volver tras comprobar que $\mathrm{K}$. W. Butzer continuaba al frente del estudio geológico y considerando, según anota LE en su diario, que "Butzer y él van a estudiar lo mismo".

El proyecto de almacén y otras dependencias planeado en 1973 -"el laboratorio"-, fue también objetivo prioritario desde los primeros días de la nueva etapa. LE anota visitas de los arquitectos el 23 de junio, el 15 de julio y el 2 de septiembre. El 21 de julio Freeman iría a Soria a recoger en el Colegio de Arquitectos el proyecto de Javier Poch Broto y Alberto Campanero García, arquitectos asociados a Antonio Almagro, también arquitecto e hijo de Almagro Basch. Pero pronto surgieron importantes problemas con uno de los dueños -Samuel Navalpotro, vecino de Miño- en relación con el precio de la finca en que se pensaba edificar. Después de muchas reuniones en las que intervino activamente LE, llegan el 1 de agosto, en busca de solución, Martín Almagro y Juan Ignacio Sáenz Diez, diputado de UCD por Soria. Almagro continuó activamente las gestiones y el 13 de agosto comunicaba que "parece que la expropiación marcha". A primeros de septiembre volvió una vez más Almagro a Ambrona, pero la campaña terminaría sin que se acabara de resolver el asunto.

Las gestiones y preparativos para levantar el nuevo museo no se detuvieron ahí. El 17 de enero de 1981 aparecen sueltos en $\mathrm{ABC}$ y en El País recogiendo que el Ministerio de Cultura había iniciado los trámites para la adquisición de todo el terreno del yacimiento, sin embargo solo se llegó a comprar el que iba a ocupar el museo en ciernes. El resto ha continuado en manos particulares originando continuos problemas que afectan a la integridad del yacimiento. En cuanto a las obras, el 24 de junio de 1981 retornan los arquitectos para discutir el emplazamiento del museo-laboratorio y renacen los problemas con las tierras, al parecer expropiadas "solo de nombre". El 1 de julio aparece de nuevo el parlamentario Juan Ignacio Sáenz Diez intentando intermediar y finalmente se consigue acuerdo para construir en la parte sur del parking.

De manera paralela se organiza la campaña de ese año. A finales de mayo Howell y Freeman se reúnen con LE para iniciar los preparativos, y como de costumbre ella asume el encargo de apalabrar la incorporación de los obreros necesarios. El 6 de junio llegan a Ambrona Freeman y Judith Odgen, el 8 Howell y Cory Woolf. Empiezan el 11, y se trabaja con normalidad los siguientes días. El 25 de junio, además de cuatro estudiantes americanos y tres ingleses -ninguno español- hay una veintena de jornaleros procedentes de Ambrona, Torralba, Guadalajara, Miño, Torrecilla del Ducado, Sigüenza, Fuencaliente, Medinaceli, y Bujarrabal. El coste de este personal alcanzó una cifra importante. Realizaron en conjunto unas 240 semanas de trabajo, que a 10.000 ptas. por semana supuso un gasto aproximado de 2.400.000 ptas. en salarios, cantidad a la que hay que añadir los costes sociales, ya que eran contratados reglamentariamente a través de la oficina de empleo.

Las operaciones se extendieron por la parte NE de la Loma de los Huesos, donde se excavaron $576 \mathrm{~m}^{2}$ repartidos en 64 cuadrículas (Fig. 11). En ese sector el nivel Va -equivalente al AS6J de la estratigrafía actual (Pérez González et al. 2005)- siguió siendo muy rico en útiles y restos de caballo y curiosamente proporcionó algún resto de elefante, género no frecuente en la unidad estratigráfica media de Ambrona. A primeros de agosto se alcanzó el Keuper en algunas cuadrículas, detectando en ellas la presencia de pavimento. Pero el 12-13 de agosto LE se enfrentaba con una estratigrafía que consideraba poco clara, con "lío de huesos", antes de alcanzar el nivel inferior. Las cuadrículas inmediatas hacia el NO continuaban dando huesos "en el pavimento y debajo de él, o lo que sea”, según anota en el diario. El 15 confirma que se trataba del pavimento, y el 24 señala que se desdoblaba en dos en el borde norte del yacimiento. El 25 Butzer está "estudiando por aquí y por allá", concluyendo que "Va es de solifluxión, luego acarreado". En todo caso las dudas con la estratigrafía continuaron. El 26 se excava "el pavimento o lo que sea" en varias cuadrículas y se abre "al fin" en la parte del parking donde se iba a construir, apareciendo restos faunísticos a tan solo $5 \mathrm{~cm}$ de la superficie. Por su parte Nick Toth y Kathy Schick "creen que excavando como excavamos se pueden pasar por alto las lascas pequeñas", optando por organizar un sistema de cribado para comprobarlo. Los problemas con la identificación del pavimento subsistieron hasta el final de la campaña: "En el último momento (el 11 de septiembre) Gary me llama para enseñarme entre P123 y P124 arena con líneas de marga debajo de las margas $\mathrm{Ph}$ IV... me ha parecido en general este año que en ningún nivel hemos llegado hasta el fondo. Les (Freeman) no estará contento, si es así quedan unos 25 cuadros por (terminar de) excavar".

A la vez que en la Loma de los Huesos se abrieron cortes en el Campo Norte, al otro lado de la carretera de acceso, donde ya se había excavado en 1963. El 17 de agosto "Clark se interesa por la loma al N" y se consigue permiso de los propietarios. "Clark quiere hacer trincheras, mientras que Les quiere hacer cuadrículas". Rod, que en ausencia de los directores debía tomar la iniciativa, no sabía a quién de los dos hacer caso, 


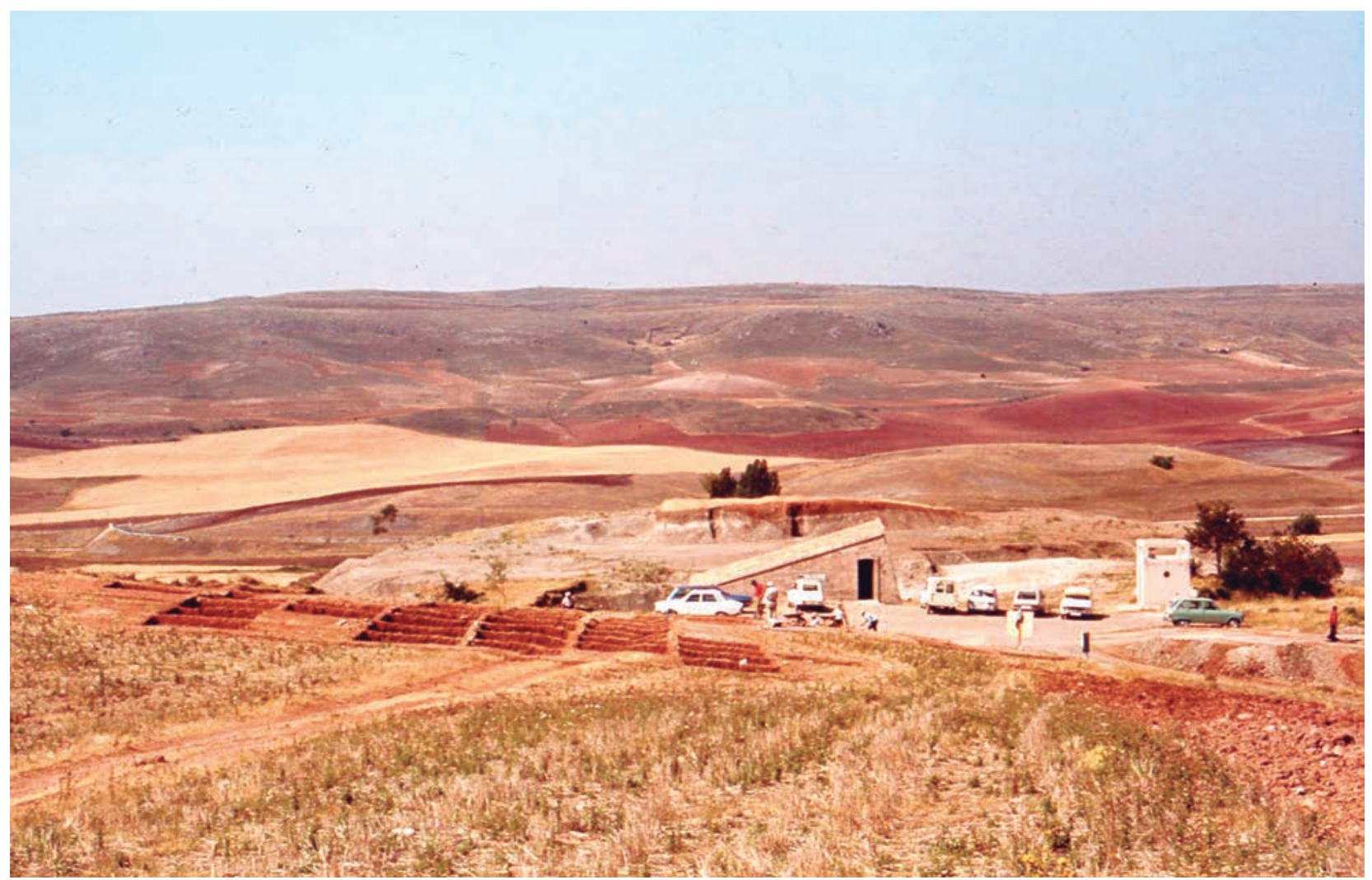

Fig. 11. Cuadrículas en excavación en el sector NE de Ambrona en 1981 (foto E. Navalpotro, vecino de Ambrona, trabajador contratado en varias campañas en Ambrona). / Grids under excavation in the NE sector of Ambrona in 1981 (photo E. Navalpotro, neighbour of Ambrona, worker hired in several Ambrona campaigns).

pero finalmente se trabajó en cuadrículas. Uno de los obreros, Jesús Riosalido, acierta a encontrar, algo más abajo de donde se había pensado que estaba, terreno excavado años atrás. LE describe la estratigrafía y los restos que van apareciendo, marga y luego arena con huesos grandes. El 20 aparece un cráneo de elefante joven y en días sucesivos el tajo Norte sigue dando materiales, prolongándose la labor en esta zona hasta el 8 de septiembre. El 17 concluyen, aunque habrá actividad hasta el 24, fecha en que regresa Howell que el 11 había viajado a California.

En la larga temporada de 1981 LE estuvo presente todo el tiempo, ejerciendo como máxima responsable en las ausencias de Howell y Freeman. Otros jóvenes técnicos que cooperaron, citados en el diario sin nombres completos a veces, fueron Roderic V. Moore -una semana de julio y otra de agosto-, Gary Richards, Deborah Peterson, Alan Sokoloff -en julio y primera quincena de agosto- y Frank Merrril algunos días de julio. Entre los seniors destaca LE la participación de Kathy Schick y Nick Toth algunos días en agosto y en septiembre. También Karl Butzer, que permaneció en la excavación del 24 de agosto al 3 de septiembre y Charles Turner, también geólogo, del 25 de junio al 10 de julio y entre el 1 y el 7 de septiembre. En relación con el estudio de la fauna fue importante la presencia de Richard
Klein los primeros 10 días de julio y al principio de septiembre, colaboración que según LE no contó con el beneplácito de Aguirre. Del estudio de la microfauna se hizo cargo Carmen Sesé, que muestreó en Ambrona a lo largo del mes de julio y algunos días en septiembre, con la colaboración de Enrique Soto. Se señala también la presencia de "un geofísico" no identificado el 3 y el 4 de septiembre, la del restaurador Juan Laguna -encargado habitual de la recogida final, embalaje y transporte de la fauna a los museos de Madrid- y la de Judith Odgen como dibujante. La lista de especialistas que atraídos por el interés que despertaba Ambrona llegaron hasta allí en 1981es muy notable, LE proporciona una relación exhaustiva con nombres y fechas. Mary Leakey llegó a mediados de junio y a los pocos días, el 23 de ese mes, el geólogo de Olduvai Richard Hay, coincidiendo con la visita de Dorothy y Jim Steim. Arthur Jelinek volvió a Ambrona el 30 de junio, Glynn Isaac el 20 y 21 de julio, Denise de Sonneville-Bordes acompañada de Prat, Debenhat y Jean Pierre Texier el 27, y en la misma fecha Paola Villa. Ofer Bar-Yosef llegó tres días después; Eric Delson el 13 de agosto y Eugène Bonifay en septiembre. Lo más selecto del elenco mundial de estudiosos del Pleistoceno y del Paleolítico antiguo se acercó a conocer los resultados que se estaban obteniendo en Ambrona. 
La construcción, por fin, del nuevo Museo probablemente determinó la tregua que se produjo en 1982. También pudo influir la ausencia de Freeman, ocupado en la excavación de El Juyo (Cantabria), circunstancia que en 1983 volvería a impedir su presencia en Ambrona. Los preparativos para una nueva campaña, que sería la última del equipo norteamericano, comenzaron a mediados de junio de 1983, ya con el reciente edificio levantado, si bien todavía vacío. En ausencia de Freeman, Clark Howell estuvo auxiliado por Gary Richards, Rod Moore y especialmente por LE en las primeras gestiones, algunas ya de entrada enfocadas a excavar en el Campo Norte, y otras a la imprescindible contratación de trabajadores, que llegarían a 14 a lo largo de la temporada. En el permiso de excavación, exclusivamente para Ambrona y fechado el 23 de junio, vuelven a aparecer como directores Almagro, Howell y Freeman, por ese orden, acogidos al patrocinio del Instituto Español de Prehistoria. De ellos ni Almagro, ni Freeman asumieron sobre el terreno tareas de dirección, labor que recayó exclusivamente en Clark Howell, relevado por LE en sus frecuentes ausencias al tener que ocuparse también de gestiones y compras.

La labor fundamental de LE en esos meses consistiría en levantar plantas de las áreas excavadas y empezar a traducir al español memorias de Torralba y de Ambrona. La documentación conservada por LE y actualmente depositada en el CENIEH, incluye los originales de dos monografías que no se han llegado a publicar. Una dedicada a Torralba, con doble versión, la primera en español fechada en 1980 (L.G. Freeman, F.C. Howell, K.W. Butzer, E. de Aguirre, R.G. Klein, T.P. Volman: Torralba: un matadero achelense en la Meseta castellana), y la segunda en inglés, más amplia, de 1988 (Torralba: an Acheulean Site on the Spanish Meseta, L. G. Freeman and F. Clark Howell, editors), con las aportaciones incluidas en el original español revisadas, más otras de algunos de aquellos autores y de Aguirre y C. Diez de Lomana, F. Prat, H.-D. Kahlke, T.P. Volman, R. Klein y K. Cruz-Uribe y A. Azzaroli.

De la segunda monografía, sobre Ambrona, el legado incluye la versión española -posiblemente traducida por LE-, titulada "Ambrona 1980-81. Excavaciones en un yacimiento de ocupación achelense del centro de España", y los autores principales son F. C. Howell, L.G. Freeman y M. Almagro Basch, con colaboraciones de E. Aguirre, K. W. Butzer, M. Hoyos (el único original que falta, probablemente nunca entregado), M. Kerney, R. Preece, H.P. Scwarcz, A. Latham, C. Sesé, F. de Borja Sanchiz, I. Doadrio, C. Turner y N. P. Toth. Las fechas de algunos capítulos indican que este original se redactó a partir de 1981, y quedaría terminado antes del fallecimiento de M. Almagro, en agosto de 1984.

El primero de los manuscritos mencionados, el de Torralba en español, se envió al Ministerio de Cultura en 1980, a propuesta de Almagro, para su publicación en Bibliotheca Praehistorica Hispana. La edición se fue retrasando y finalmente surgieron problemas en el pro- ceso de impresión que llevaron a los autores a retirar el original en 1985. El documento conservado por LE corresponde a una primera versión con correcciones aparentemente de su mano, que se incorporaron al texto posteriormente.

La campaña de 1983 fue subvencionada, como las dos precedentes, por la NSF y el Comité Conjunto Hispano-Norteamericano para Asuntos Educativos y Culturales, entidades a las que se añadió la National Geographic Society. Algunas anotaciones de LE ayudan a conocer el alcance del presupuesto manejado, del orden de 5 millones de pesetas, muy por encima de lo habitual en las excavaciones españolas de la época. Los obreros contratados - 16 según las notas de LE- percibían 2.400 ptas cada uno de los 5 días laborables de la semana, lo que teniendo en cuenta el tiempo de contratación -unas 130 semanas totales- supuso un importe aproximado de 1.560 .000 ptas más costes sociales. El uso del tractor y la pala ascendía a 2.500 ptas por hora trabajada, disponer de la parcela en que se ubicaba la excavación del Campo Norte costó 10.000 ptas y una cantidad comparable supuso la intervención en la Loma de los Huesos. El trabajo de los técnicos estaba retribuido con 5.000 ptas diarias -el 1 de julio LE anotaba "cobro 75.000 ptas por medio mes"-, a todo lo cual hay que sumar gastos de viajes, alojamiento y manutención durante toda la campaña.

El 20 de junio se excavaba en el Campo Norte (Fig. 12). Rod y Gary se ocupaban de los problemas de conexión con los niveles de la Loma de los Huesos, partiendo de suponer que existían correspondencias estratigráficas. En las escasas referencias encontradas en el diario de Echaide, los depósitos del Campo Norte se entendían integrados en la "Formación Torralba", si bien dentro de un sistema de drenaje distinto al representado en la Loma de los Huesos. Se trabajaba inicialmente en cinco cuadrículas y en una de ellas había aparecido según las notas de LE un estupendo bifaz en un nivel de "gritty sand". Cuando se bajaba mucho -en algún caso hasta 2,4 m de profundidad- sin encontrar más que marga se abandonaban, siguiendo solo cuando aparecían arenas. Se registraron en todo caso pocos restos, entre los que destacaba un cráneo de bóvido. La industria bifacial de aquella zona, al menos 3 bifaces antes y otro en esta campaña, se llegó a interpretar como más evolucionada que la de la "ocupación inicial" de la Loma de los Huesos (Howell, 1982). El 1 de julio se empezó a despejar con la pala por el "oeste, noroeste y algo de norte", con la intención de continuar excavando por allí.

En la Loma todo el personal disponible trabajaba a primeros de julio entre los dos museos, donde según LE iba quedando una bonita superficie de la que no se sacaban los restos que aparecían, pensando en la visita de los reporteros de la National Geographic. El 5 de julio se terminó de limpiar en el Campo Norte y todo el personal pasó al yacimiento clásico, unos al sector entre los museos y otros abriendo nuevos cuadros en 


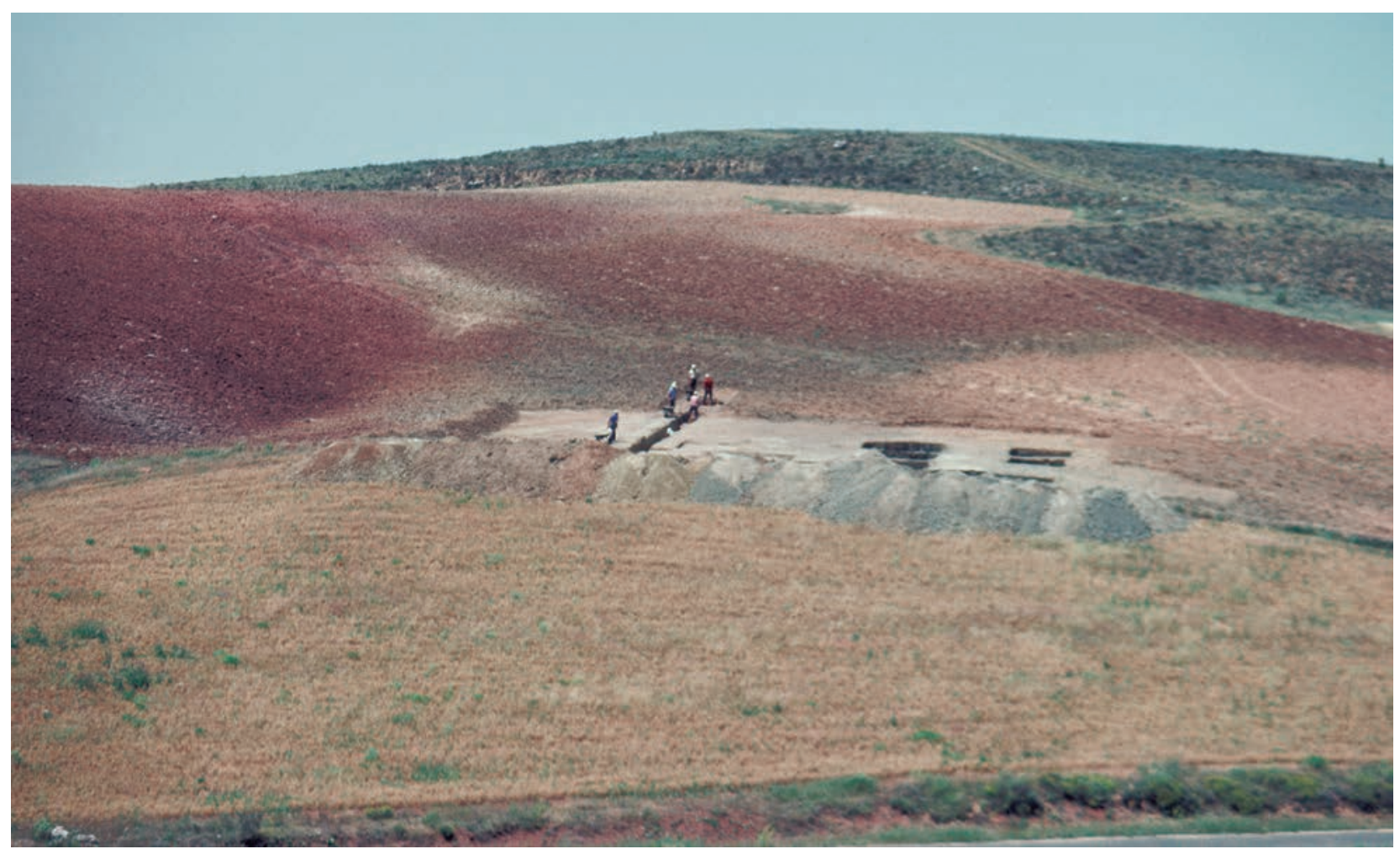

Fig. 12. Vista del área de excavación en el Campo Norte en la campaña de 1983 (Archivo Echaide. CENIEH). / View of the excavation area in the North Field, 1983 campaign (Echaide File, CENIEH).

la ocupación media, al sur del área excavada en 1980. El día 11 se empezó una nueva trinchera en la parte Norte (Fig. 12), "los veteranos al Norte, a hacer una trinchera Norte-Sur hasta llegar al Keuper o al pavimento"..., "los demás en sus cuadros en la parte este de la Loma hasta alcanzar el pavimento". Desde el día 13 al 18 Howell y los componentes americanos del equipo se van a Francia a visitar las excavaciones de L'Arago, y LE queda encargada de todo. El 14 escribe que "en la Trinchera $\mathrm{N}$ me parece que todos están en Keuper", y manda limpiar 3 cuadros más en dirección $\mathrm{N}$ porque parecía verse pavimento como a $25 \mathrm{~cm}$ de la superficie. Aparece un bifaz en plena marga y en algún cuadro se llega al pavimento. El 15, antes de la vuelta de Francia de Howell y acompañantes, aparece una gran mancha "ocre y negra" entre las margas que llama considerablemente la atención. A la vuelta de Francia, el 18, todo el mundo quiere ver el bifaz y opinar sobre la mancha de ocre, muy enigmática en el contexto de Ambrona, que finalmente llega a ser interpretada como "brecha de cueva, con huesitos dentro".

El 30 de agosto se da por finalizada la campaña, que contó como la anterior de nutrida colaboración técnica no española, con LE como excepción más destacada y junto a ella solamente Chus Riosalido, oriunda de la comarca y contratada en julio y agosto para ayudar en el registro de restos. Blanca Izquierdo residía en Ambrona, pero ya no participaba en los trabajos.
La asistencia de Roderic V. Moore y de Gary Richards durante todo el tiempo fue fundamental, y también contribuyeron de manera importante Alan Sokoloff en julio, así como Richard y Kathy Klein del 13 al 19 de agosto. Junto a ellos 9 obreros todo el tiempo y otros 7 en períodos de 1 a 2 meses, la mayoría expertos de años anteriores (Fig.13). Los visitantes no alcanzaron niveles precedentes, destacando miembros de la familia Ghetty -de los que se intentaba captar recursos económicos-, o los enviados de la NGS, que realizaron un completo reportaje empleando incluso medios aéreos.

Aunque lo previsto era continuar, así finalizó la intervención norteamericana en Ambrona. LE recogía el 13 de julio palabras de Howell comentando que al año siguiente, 1984, no habría excavación pero que se seguiría en 1985. Si esa era en efecto la intención, aunque no lo podamos saber con certeza, posiblemente se vio afectada por los cambios administrativos que experimentó en España la gestión de la Arqueología, al ser transferidas a las Autonomías en septiembre de 1983 prácticamente todas las competencias. El fallecimiento de Martín Almagro, el 28 de agosto de 1984, que se había encargado de resolver en España las formalidades administrativas, pudo ser otro factor clave en tan precipitado desenlace. Es posible que el ignorado paradero de la industria lítica de la última campaña de Ambrona, que tendría que estar en el Museo Numantino, tenga que ver con un final no programado y un tanto abrupto. 


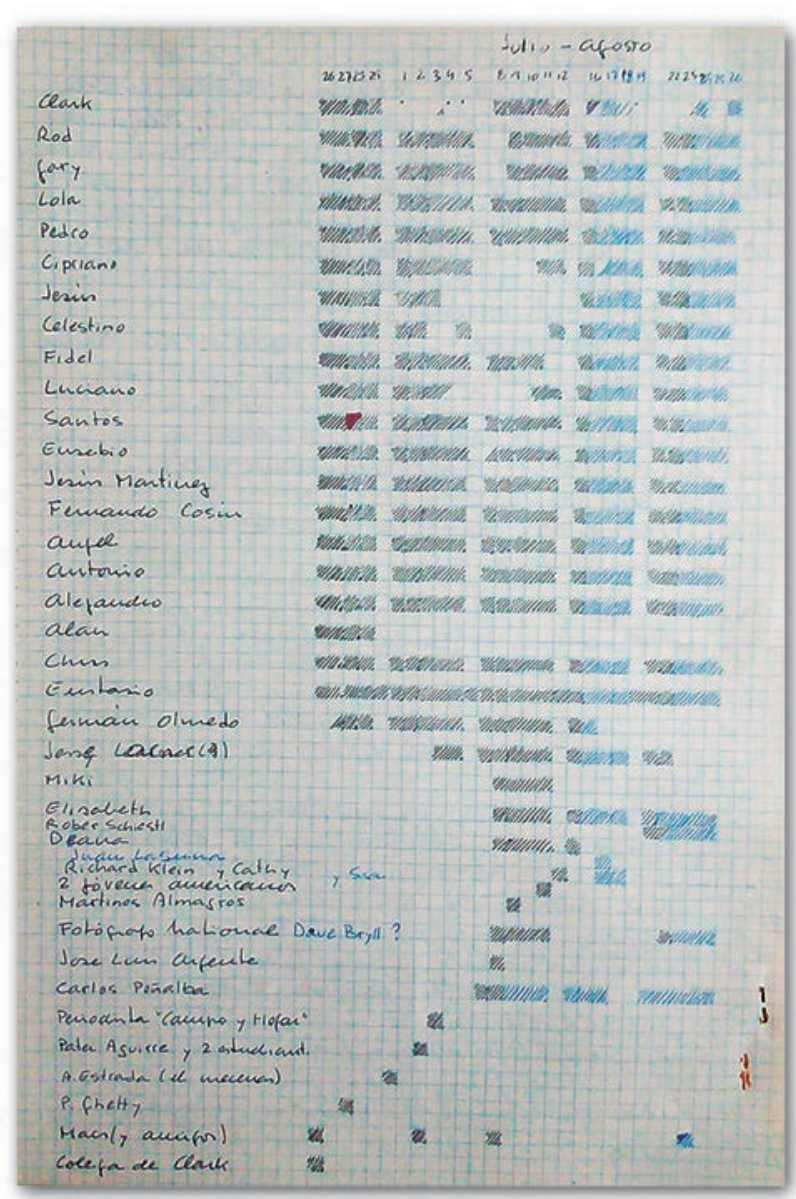

Fig. 13. Tipo de estadillo de control que llevaba LE de las fechas trabajadas en Ambrona por los miembros del equipo de investigación y por los obreros contratados (el reproducido va del 26 de julio al 26 de agosto de 1983). Al final incorpora también nombres y fechas de visitantes (Archivo Echaide. CENIEH). / Ambrona shifts register of the research team and the contracted workers (this one goes from July 26 to August 26, 1983). Visitor's names and dates are also incorporated in the end (Echaide File, CENIEH).

\section{REFLEXIÓN FINAL A MODO DE CONCLUSIÓN}

El proyecto que inició Howell en 1961 en Torralba, continuado por él mismo en 1980 en Ambrona junto a su discípulo Leslie G. Freeman, en el que LE participó plenamente desde el comienzo, adquirió de inmediato una importancia análoga a la de los más punteros del ámbito internacional en el campo del Paleolítico antiguo. En 1961 Howell formó un equipo interdisciplinar que trabajó con una perspectiva paleoantropológica integral totalmente innovadora. Torralba y Ambrona llegaron a ser referencias clave en los cimientos de una corriente de investigación sobre las primeras etapas de la humanidad que continua vigente en nuestros días. Lamentablemente, en la década de 1960 la Arqueología española carecía de grupos interesados en el paleolítico antiguo capaces de asimilar y aplicar los avanzados planteamientos teóricos introducidos por Howell. Tan solo cabría recordar los ensayos de Emiliano Aguirre en Gándaras de Budiño, que no prosperaron; la te- sis iniciada por LE en ese enclave gallego pudo haber supuesto una continuidad interesante, pero el proyecto no llegó a cuajar. Después de 1983 la relación de Dolores Echaide, que ya en 1973 se había considerado "ex prehistoriadora", con la arqueología paleolítica fue decayendo. Estaba claro el peso de su vinculación con Howell, con Freeman y con el proyecto de Ambrona, y paralelamente su falta de integración en cualquier marco español relacionado con la investigación arqueológica. Más allá de la participación de Dolores Echaide en la investigación de Torralba y Ambrona, la historia de estas intervenciones pone de relieve la escasa influencia de unos proyectos tan destacados, especialmente el inicial, en el desarrollo de los estudios sobre Paleolítico inferior en España.

\section{AGRADECIMIENTOS}

Para realizar este trabajo han sido imprescindibles los diarios y otros originales elaborados por Dolores Echaide. Su hermana, Ana Echaide, ha organizado toda esta documentación y la ha depositado en el CENIEH, donde se conserva a disposición de los interesados en su consulta. Los textos en inglés (abstract y leyendas de figuras) han sido revisados por Marta Muñiz Pérez. Expresamos a ambas nuestro sincero agradecimiento, así como a dos revisores anónimos y a Álvaro Arrizabalaga, cuyas sugerencias han contribuido de manera eficaz a la forma final de nuestro original. Este trabajo se ha realizado en el marco del proyecto PGC2018093612-B-I00, concedido por el Ministerio de Ciencia, Innovación y Universidades, y del que son IP's Susana Rubio Jara y José Yravedra Sainz de los Terreros.

\section{BIBLIOGRAFÍA}

Aguirre, E. de, 1964. Las Gándaras de Budiño. Excavaciones Arqueológicas en España, 31. Ministerio de Cultura. Madrid.

Aguirre, E. de, 2005. Torralba y Ambrona. Un siglo de encuentros. Zona Arqueológica 5, 40-77.

Echaide, M. D., 1971. La industria lítica del yacimiento de Budiño (Pontevedra). Munibe, XXIII(1), 125-154.

Howell, F.C., Butzer, K.W., Aguirre, E. de, 1962. Noticia preliminar sobre el emplazamiento achelense de Torralba. Excavaciones Arqueológicas en España, 10. Ministerio de Cultura. Madrid.

Howell, F.C., 1965. El yacimiento achelense de Ambrona. Noticiario Arqueológico Hispánico VII (1-3), 7-23.

Howell, F.C., Freeman, L. G., 1982. Ambrona: an early Stone Age site on the Spanish Meseta. The L. S. B. Leakey Foundation News 22, 1113.

Pérez-González, A., Santonja, M., Benito, A., 2005. Secuencias litoestratigráficas del Pleistoceno medio del yacimiento de Ambrona. Zona Arqueológica 5, 176-189.

Straus, L.G., 2016. The Chicago connection in Spanish Paleolithic Prehistory. In: Delley, G., Diaz-Andreu, M., Djindjian, F. et al. (Coords.), History of Archaeology: International perspectives, 111-119. Archeopress, Oxford. 